\title{
ON SYNCHROTRON RADIATION SPECTRUM IN CLASSICAL AND QUANTUM MECHANICAL LIMITS
}

\author{
F. SCarlat and N. Baboi \\ National Institute for Laser, Plasma and Radiation Physics \\ Electron Accelerator Laboratory \\ P.O. Box MG-6, Bucharest, 76900 Romania
}

\begin{abstract}
The spectrum of the synchrotron radiation called "beamstrahlung" is an universal function of the photon energy divided by the parameter $E_{c}$ called "critical energy". This paper presents the spectrum for both the classical regime, when the electron beam energy, $E$, is bigger than the critical energy, and the quantum regime, when the beam energy is less than the critical energy. Results of the calculations show that, in the latter case, less beamstrahlung is emitted than with the classical one.
\end{abstract}

PACS numbers: 41.60.Ap, 03.65.-w

From the first observations of the radiation emitted by high-energy electrons in a constant magnetic field, people looked for applications in various domains, mostly in solid state physics. For this purpose, the properties of this synchrotron radiation were studied both theoretically and experimentally. As regards the theoretical aspect, the calculations made by Schwinger [1] in 1949 are still used as a reference even today, after 50 years.

Schwinger's formulas for the radiated power, deduced in a classical approximation, are applicable in most of the practical interest cases. However, the new facilities with very high energy electron beams (such as beam-beam colliders), as well as the new perspectives opened in science lead us towards zones where the classical relations are no longer valid and quantum mechanical corrections have to be made.

In this paper, the difference between the classical results and the quantum ones, corresponding to very high energies and low path radii of the electron, is discussed. The domain of energy and radius where the classical approximation is valid and the one where quantum corrections become very important are highlighted.

According to the classical approximation, the total radiated power, $P$, emitted by an electron in uniform circular motion is given, by [1]:

$$
P=\frac{2}{3} \omega_{0} \frac{e^{2}}{R}\left(\frac{E}{m_{0} c^{2}}\right)^{4},
$$


where $E$ is the electron energy, $R$ is the radius of the trajectory, $\omega_{0}=c / R$ is the rotational frequency in the orbit, $c$ is the light velocity, $m_{0}$ is the electron rest mass, and $e$ is the electron charge.

Several authors (e.g. [2-5]) made quantum calculations or discussed the applicability limits of relation (1). Realistic enough seem to be the calculations of Schwinger [2], subsequent works generally confirming or adding superior order corrections.

The first-order quantum corrections to the total radiated power, $\delta P$, is

$$
\delta P=3^{1 / 2} \frac{55}{36} \omega_{0} \frac{e^{2}}{R}\left(\frac{E}{m_{0} c^{2}}\right)^{4} \frac{\hbar \omega_{\mathrm{c}}}{E},
$$

where $\hbar=h / 2 \pi$ is Planck's constant, and $\omega_{\mathrm{c}}=3 / 2 \omega_{0}\left(E / m_{0} c^{2}\right)^{3}$ is the critical frequency.

While speaking of radius, we implicitly think of magnetic field, because usually there is a magnetic field bending the electron trajectory. That is why synchrotron radiation is sometimes called "magnetic bremsstrahlung". Large fields involve small radii.

An often used parameter to describe the quantum nature of the process of photon emission by the electrons in magnetic field (beamstrahlung) is

$$
r=\frac{E}{m_{0} c^{2}} \frac{B}{B_{\mathrm{c}}},
$$

where $B$ is the magnetic field strength, and $B_{\mathrm{c}}=m_{0}^{2} c^{2} / e \hbar=4.42 \times 10^{9} \mathrm{~T}$ is the Schwinger critical field strength. In the case of colliders, $B$ is the average electromagnetic field seen by a particle while traversing the opposite bunch. For values of $\Upsilon$ up to about 0.01 (sometimes even bigger), the classical approximation can still be used. But for greater values it is advisable to use quantum relations.

In fact, up to a constant, the parameter $Y$ is the same with the relative quantum correction, $\delta P / P$, which justifies the use of $\Upsilon$ as a measure of applicability of the classical approximation.

In Fig. 1, the domain of applicability of the classical results and the one where quantum effects should be taken into account are shown. For bigger $\Upsilon$, higher order corrections than relation (2) should be made.

It is interesting to see how the spectral power modifies in the quantum approximation with respect to the classical one. In the classical regimes, the spectral power, $\mathrm{d} P / \mathrm{d} \omega$, emitted by an electron in magnetic field is [1]:

$$
\frac{\mathrm{d} P}{\mathrm{~d} \omega}=\frac{3^{3 / 2}}{4 \pi} \frac{e^{2}}{R}\left(\frac{E}{m_{0} c^{2}}\right)^{4} \frac{\omega_{0} \omega}{\omega_{\mathrm{c}}^{2}} \int_{\omega / \omega_{c}}^{\infty} K_{5 / 3}(x) \mathrm{d} x,
$$

where $\omega$ is the photon frequency, and $K_{5 / 3}$ is the modified Bessel function.

The fraction of $\mathrm{d} P / \mathrm{d} \omega$, which depends on $\omega$, can be drawn as the function

$$
S\left(\frac{\omega}{\omega_{\mathrm{c}}}\right)=\frac{\omega}{\omega_{\mathrm{c}}} \int_{\omega / \omega_{\mathrm{c}}}^{\infty} K_{5 / 3}(x) \mathrm{d} x .
$$

This actually gives the emitted radiation spectrum.

Classically, if the beamstrahlung photon frequency is measured in units of the critical energy, the spectrum is like the one represented by the solid line in Fig. 2. 


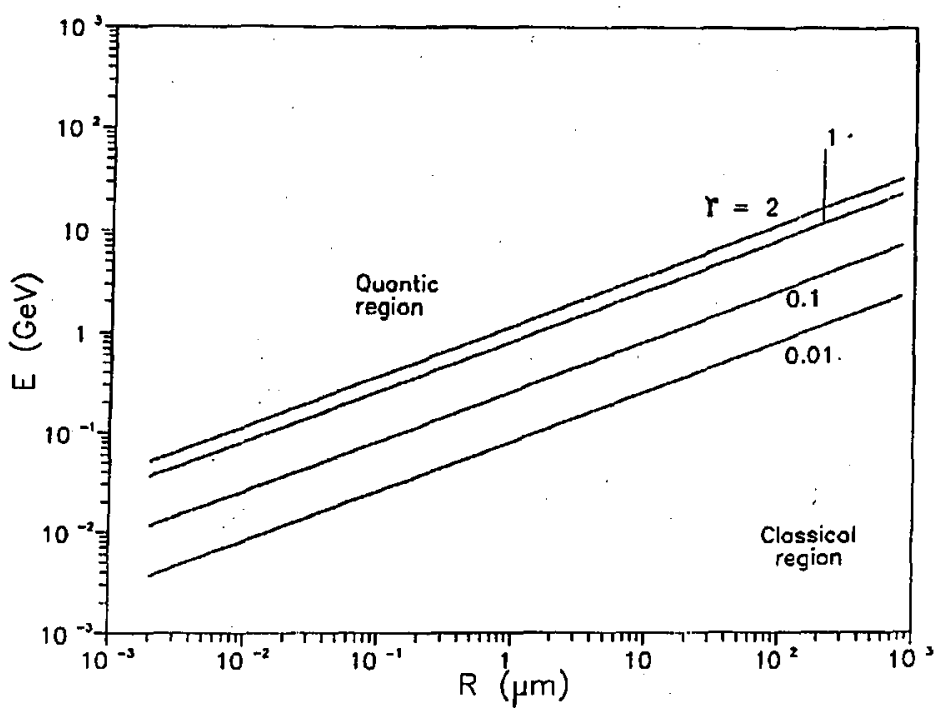

Fig. 1. The domains of validity for the classical regimes and the quantum one, respectively.

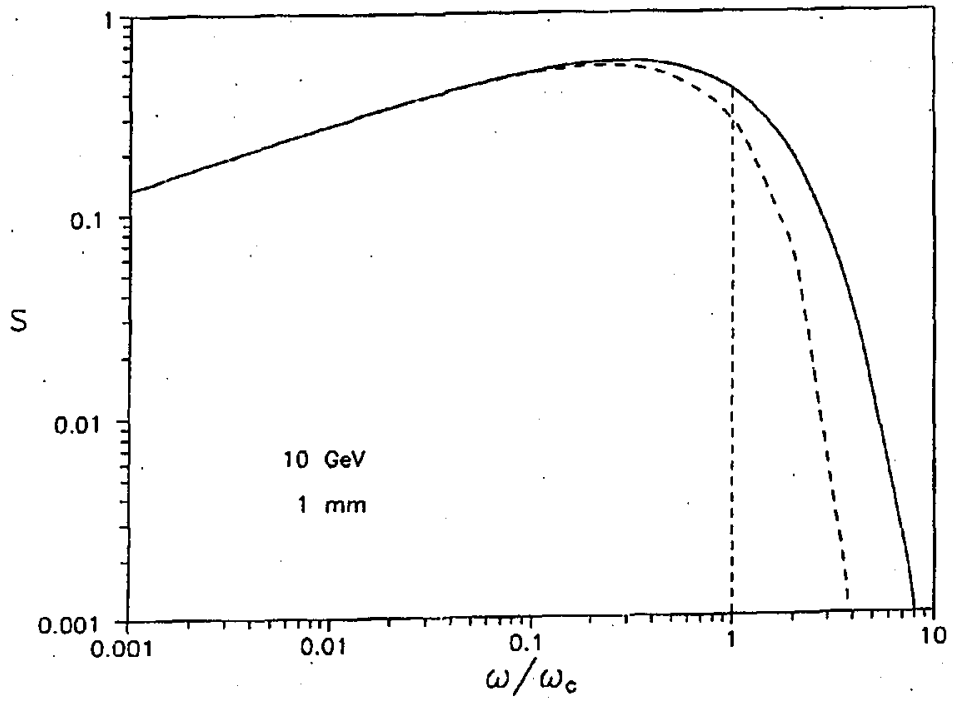

Fig. 2. The spectra for $E=10 \mathrm{GeV}$ and $R=1 \mathrm{~mm}$ for the cases: classical approximation (solid line), and quantum approximation (dashed line).

Instead of frequency, on the horizontal axis we may place the photon energy, $E_{\gamma}$, divided by the critical energy $E_{\mathrm{c}}=\hbar \omega_{\mathrm{c}}$, since $\omega / \omega_{\mathrm{c}}=E_{\gamma} / E$. The critical energy splits the spectrum into two equal parts regarding the total radiated power.

The classical spectrum is good as long as the electron energy divided by the 
critical energy is much greater than one. When $E / E_{\mathrm{c}}<1$, the classical spectrum is no longer valid because energy conservation would be violated. A small ratio $E / E_{\mathrm{c}}$ means very high electron energies and very small path radii (or, equivalent, very large magnetic fields). In this case, less beamstrahlung is emitted than the classical Eq. (4) involves.

The results of the quantum calculations show that the emitted spectral power differs from 4 only by the function $S$. The quantum $S_{q}$, in the first order approximation, is given by

$$
S_{q}\left(\frac{\omega}{\omega_{\mathrm{c}}}\right)=\frac{\omega}{\omega_{\mathrm{c}}} \int_{\omega / \omega_{\mathrm{c}}(1+\hbar \omega / E)}^{\infty} K_{5 / 3}(x) \mathrm{d} x
$$

This function is shown in the same Fig. 2, by the dashed line, for an energy of $10 \mathrm{GeV}$ anc a radius of $1 \mathrm{~mm}$. It can be seen that, for low photon frequencies, the two curves are practically identical, while for higher $\omega, S_{q}$ is smaller than $S$. In other words, a shift of the spectrum towards lower frequencies takes place. In the same time, $\omega_{\mathrm{c}}$ (or $E_{\mathrm{c}}$ ) loses its classical meaning of dividing the spectrum into two equal parts. Less beamstrahlung is produced because of the quantum effects.

For the case shown in Fig. 2, the per cent correction to the total radiated power is of the order of $200 \%$.

In the electron-positron colliders (energies of $\mathrm{GeV}$ up to $\mathrm{TeV}$ ), the particle trajectory is bent by the electromagnetic field of the opposite bunch $[6,7]$. The radius of the trajectory decreases while the energy increases.

The quantum effects are also very important in the perspective of producing synchrotron radiation with the help of solid structures. When a thin electron beam passes through a crystal, the particles will have an oscillatory path under the Coulomb field of the ionic nodes of the structure. The instantaneous electron radii may be of the order of or less than the distance between the crystalline lattice planes which is, generally, of the order of $\AA$. In this case, the quantum correction will be much greater, of the order of $10^{2}, 10^{3}$. But, of course, higher order quantum corrections should be applied and these will further limit the radiated power.

Moreover, in the case of the free electron laser, operating in the short wavelength regions (VUV, X-regime) for example, for high-energy electron beam and electromagnetic undulator, the critical energy of synchrotron radiation may be bigger than the electron beam energy. For a radius $R$ equal to the betatron oscillation amplitude $a\left(=\lambda_{\mathrm{u}} K / 2 \pi \gamma_{0}\right)$, where $\lambda_{\mathrm{u}}=2 \gamma_{0}^{2} \lambda, \lambda=10^{-10} \mathrm{~m}$, for a beam energy $E=1 \mathrm{GeV}$, the critical energy has the value of $E_{\mathrm{c}}[\mathrm{eV}]=2218 E^{3}[\mathrm{GeV}]$ $/ R[\mathrm{~m}]=35.6 \mathrm{GeV} \gg E$.

In this way, the Renieri classical limit condition is reconfirmed which asserts that the spread in an electron energy wave function at the end of the undulator length is smaller than the wavelength of the ponderomotive potential, $\lambda_{\mathrm{pm}}$, namely: $\lambda_{\mathrm{pm}}^{2}>\lambda_{\mathrm{c}} L_{\mathrm{u}} / \pi \gamma_{0}^{3}$, where $\lambda_{\mathrm{c}}$ is the Compton wavelength and $L_{\mathrm{u}}$ is the undulator length [8].

\section{References}

[1] J. Schwinger, Phys. Rev. 75, 1912 (1949).

[2] J. Schwinger, Proc. Natl. Acad. Sci. USA 40, 132 (1954). 
[3] A.A. Sokolov, N.P. Klepikov, I.M. Ternov, Dokl. Akad. Nauk SSSR 89, 665 (1953).

[4] T. Erber, Rev. Mod. Phys. 38, 626 (1966).

[5] N.P. Klepikov, N.P., Zh. Eksp. Teor. Fiz. 26, 19 (1954).

[6] B. Richter, presented at the 2nd Int. Workshop on Laser Acceleration of Particles, Los Angeles 1985, SLAC-PUB-3630, p. 1.

[7] R.J. Noble, Nucl. Instrum. Methods Phys. Res. A 256, 427 (1987).

[8] G. Datolli, A. Reinieri, in: Laser Handbook, Vol. 6, Eds. W.B. Colson, C. Pellegrini, A. Reinieri, Elsevier, North-Holland, Amsterdam 1990. 